Recherches en histoire de l'art, histoire des civilisations, archéologie, anthropologie et muséologie

8 | 2016

Cahiers 8

\title{
Le Musée Rodin de 2015 : choix muséographiques
}

\section{Dominique Brard}

\section{OpenEdition \\ Journals}

Édition électronique

URL : http://journals.openedition.org/cel/335

DOI : $10.4000 /$ cel.335

ISSN : 2262-208X

Éditeur

École du Louvre

\section{Référence électronique}

Dominique Brard, «Le Musée Rodin de 2015 : choix muséographiques », Les Cahiers de l'École du

Louvre [En ligne], 8 | 2016, mis en ligne le 28 octobre 2016, consulté le 17 septembre 2019. URL

http://journals.openedition.org/cel/335; DOI : 10.4000/cel.335

\section{(c) (i) (3)}

Les Cahiers de l'École du Louvre sont mis à disposition selon les termes de la licence Creative Commons Attribution - Pas d'Utilisation Commerciale - Pas de Modification 4.0 International. 


\section{Cahiers de l'École du Louvre}

recherches en histoire de l'art, histoire des civilisations archéologie, anthropologie et muséologie

Numéro 8. Mai 2016

Le Musée Rodin de 2015 :

choix muséographiques.

Dominique Brard

Article disponible en ligne à l'adresse :

http://www.ecoledulouvre.fr/cahiers-de-l-ecole-du-louvre/numero8-mai2016/Brard.pdf

Pour citer cet article :

Dominique Brard, « Le Musée Rodin de 2015 : choix muséographiques. », Cahiers de l'École du Louvre. Recherches en histoire de l'art, histoire des civilisations, archéologie, anthropologie et muséologie [en ligne] nº 8, mai 2016, p. 20 à 28.

\section{cccreative \\ (B) $\ominus$}

(c) École du Louvre

Cet article est mis à disposition selon les termes de la Licence Creative Commons Attribution - Pas d'utilisation commerciale - Pas de modification 3.0 non transposé. 


\section{Cahiers de l'École du Louvre}

recherches en histoire de l'art, histoire des civilisations archéologie, anthropologie et muséologie

\section{Numéro 8. Mai 2016}

\section{Sommaire}

\section{Éditorial}

\section{Articles}

Malcolm Baker, Distinguished Professor of the History of Art, University of California, Riverside Writing about displays of sculpture: historiography and some current questions....

Catherine Chevillot, Directrice du Musée Rodin

Les enjeux d'une rénovation.

Dominique Brard, Architecte

Le Musée Rodin de 2015 : choix muséographiques

p. $20-28$

Geneviève Bresc-Bautier, Conservatrice générale du patrimoine honoraire Exposer la sculpture au Louvre au temps de Rodin, 1860-1914 p. $29-40$

Astrid Nielsen, Conservatrice des collections de sculpture, Albertinum, Dresde Exposer la sculpture dans l'Albertinum de Dresde : passé, présent et futur.....p. 41-50

Colin Lemoine, Historien de l'art, responsable des sculptures au musée Bourdelle

Amélie Simier, Conservatrice en chef du patrimoine, directrice des musées Bourdelle et Zadkine

La sculpture sur le bout des doigts. Retour sur l'élaboration

d'une salle pédagogique et tactile au musée Bourdelle.....

Cecilia Hurley-Griener, Docteur en histoire de l'art (HDR), équipe de recherche, École du Louvre

Putting sculpture on show/Exposer la sculpture : conclusion. p. $58-65$ 


\section{Dominique Brard}

\section{Le programme}

L'opération de l'hôtel Biron au Musée Rodin porte sur des travaux de toutes natures, menés par deux maîtrises d'œuvre :

- des travaux de fonctionnalités, mise aux normes, confort, et muséographie par l'Atelier de l'île architectes et muséographes,

- des travaux de gros ouvre et de restauration par l'architecte en chef des monuments historiques, Richard Duplat ACMH.

À partir du nouveau programme scientifique élaboré par le musée, la partie du travail la plus visible et la plus importante concerne la muséographie. Il s'agit de développer, en coordination étroite avec la directrice et la conservation du musée et à destination d'un large public, une image et une présentation des œuvres renouvelées, tant en contenu qu'en organisation, en mobilier et en éclairage, facilitant la compréhension de l'œuvre d'Auguste Rodin, de son histoire et de ses techniques.

Le programme muséographique comprend :

- la remise en valeur des parquets et des plafonds des salles par Richard Duplat $\mathrm{ACMH}$,

- la remise en état des murs et leur doublage pour l'accrochage des tableaux, leur mise en peinture et en couleurs,

- un nouveau mobilier muséographique,

- un nouvel éclairage muséographique,

- la signalétique muséographique et fonctionnelle par Intégral Concept.

Le programme fonctionnel comprend :

- la remise en conformité et en sécurité/sûreté du bâtiment (dont un nouveau PC de sécurité), de nouveaux réseaux électriques courants forts et faibles,

- les aménagements de confort de visite (vestiaires, sanitaires...) et d'accessibilité (ascenseur),

- des espaces logistiques et de fonctionnement interne (sanitaires, vestiaires, gardiennage et bureaux),

- des bureaux dans les combles.

\section{Les données}

Les travaux ont concerné pour la partie publique le rez-de-chaussée et l'étage, et pour les parties de fonctionnement interne les combles, les entresols, et partiellement le sous-sol.

La superficie totale de l'hôtel Biron est de $2769 \mathrm{~m}^{2}$. Le circuit de visite se déroule suivant 18 salles d'exposition et une galerie dédiée aux dessins et aux photographies, sur $1241 \mathrm{~m}^{2}$. Près de 600 œuvres sont exposées.

Principales dates :

- mi-2010 : concours de maîtrise d'œuvre

- mi-2010 à fin 2011 : études

- fin 2011 : appels d'offres entreprises bâtiment

- début 2012 à janvier 2014 : travaux phase 1 au rez-de-chaussée et premier étage moitié Ouest

- fin 2014 et début 2015 : appels d'offres entreprises muséographie

- de mai 2014 à février 2015 : réaménagement des bureaux dans les combles

- de février 2014 à juin 2015 : travaux phase 2 au rez-de-chaussée et premier étage moitié Est 
- de février 2015 à septembre 2015 : muséographie définitive de l'hôtel Biron

- novembre 2015 : inauguration

Le budget s'élève à 15,5 M€ TDC, dont 1,6 M€ alloué à la muséographie.

\section{La posture}

La caractéristique la plus singulière de l'hôtel Biron est d'être, au-delà d'un musée, le lieu où Auguste Rodin a travaillé, vécu, où lui-même a exposé son travail et ses collections; cette prise en compte va influencer fondamentalement les partis-pris de présentation et de mise en scène des œuvres.

Le projet architectural et muséographique s'est construit suivant trois axes de réflexion :

- garder l'esprit et la spécificité du musée, celui d'une demeure privée, un hôtel particulier du XVIII ${ }^{\mathrm{e}}$ siècle. Le projet préserve ce qui fait l'identité du lieu, sa magie, et les fondamentaux que sont les relations extérieurintérieur, la lumière naturelle, les vues et reflets, et surtout l'intimité, mais aussi ses défauts comme les décors partiels, les pentes des planchers, les traces des états précédents.

- développer un principe de contemporanéité, sans pastiche et tout en subtilité, par des interventions non intrusives, comme une strate de plus dans l'histoire de l'hôtel, celle d'un musée moderne, respectant le lieu avec ses qualités et ses défauts.

- viser l'excellence, à la hauteur de la collection, de la renommée du musée et de sa fréquentation.

\section{Les travaux de restauration}

L'architecte en chef des monuments historiques a principalement eu en charge quatre éléments fondamentaux indispensables à la réalisation du projet: les parquets et sols marbrés, les plafonds, les fenêtres et enfin, la cour d'honneur.

Les parquets Versailles existants et fortement dégradés ont été déposés, restaurés et reposés, tandis que certaines salles ont vu leur parquet standard remplacé par un parquet Versailles neuf. Cette opération aura aussi et permis de redonner aux planchers de l'étage la portance suffisante par rapport aux normes des établissements recevant du public et par rapport à la présence d'œuvres lourdes en renforçant les poutraisons et en réalisant des dalles de répartition en bois, et pour certaines salles en béton.

Les sols en marbre ont été réparés et complétés suivant les cas.

Les plafonds ont été restaurés, et une grande part des corniches décoratives ont été soit restaurées soit restituées.

Les grandes baies vitrées de l'hôtel, fortement dégradées, ont été restaurées ou remplacées.

Le parvis de l'hôtel et son accessibilité ont été remaniés au bénéfice des personnes à mobilité réduite. Faisant suite à la décision de modifier l'accès au musée par la cour, le portail et la clôture du côté de la rue de Varenne ont été restaurés.

\section{Le circuit et le confort de visite}

Le projet développe sur deux niveaux un circuit de visite renouvelé, calé sur le nouveau propos scientifique, chronologique en rez-de chaussée et chronothématique à l'étage.

Celui-ci se déroule de manière continue suivant deux boucles dans le même sens au rez-de-chaussée et à l'étage (par la suppression des culs-de-sac des anciens bureaux à l'étage). Simple et lisible, il favorise la compréhension du travail 
d'Auguste Rodin, le repérage du visiteur et la fluidité de la visite. Il permet également de mieux gérer la densité du public dans les salles.

La réorganisation permet d'assurer également un meilleur confort pour le visiteur (ascenseur, sanitaires, élargissement de certains passages, etc.) et une bonne sécurité (issues et escaliers de secours, etc.). L'ensemble est accessible aux personnes à mobilité réduite.

\section{Les principes muséographiques}

Le projet muséographique se fonde sur quatre principaux axes de mise en scène.

\section{Présenter la sculpture}

Parce que c'est un musée mais aussi le lieu de vie et de travail l'artiste, et surtout le lieu où lui-même exposait ses œuvres, l'esthétique du mobilier muséographique ne pouvait être anonyme, banale, ou encore hors site. Elle a été pensée dans le projet en référence aux sellettes du sculpteur, supports de présentation qu'Auguste Rodin utilisait lui-même pour présenter ses ouvres dans ce même lieu (fig. 1). Le mobilier a donc été conçu et dessiné en référence à celles-ci, mais sans pastiche, tout en minimalisme et en discrétion :
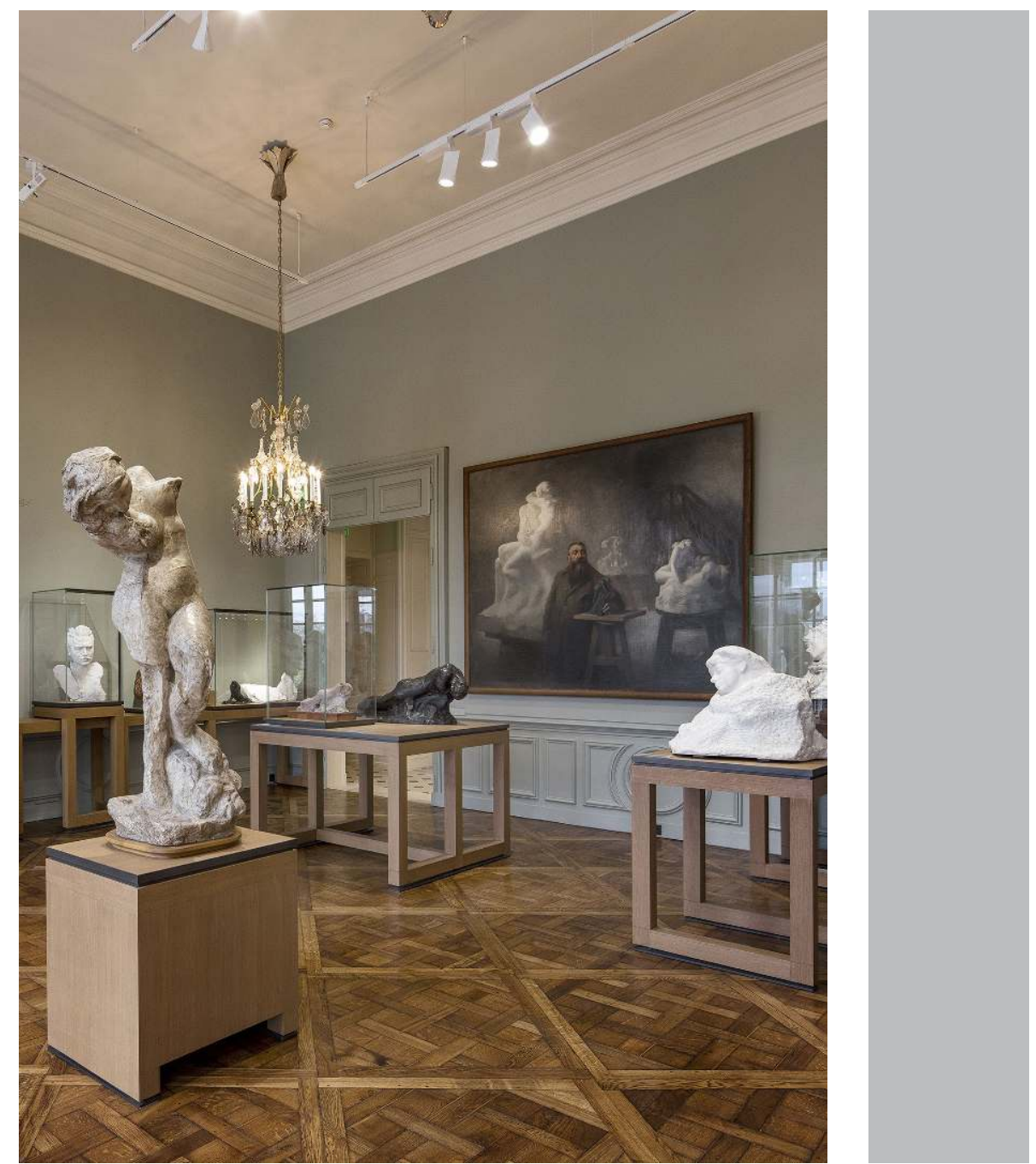

Vue de la salle 15 «Agrandissement et fragmentation » Paris, Hôtel Biron (c) Patrick Tournebœuf / OPPIC / Tendance Floue

- ce sont des supports ouverts, qui laissent passer le regard, et le parquet " continue » sous le socle,

- en chêne, ils entrent en symbiose avec le parquet et les boiseries en chêne, évitant l'introduction d'un matériau supplémentaire dans les salles,

- leur modénature montre un assemblage de traverses de bois assemblées, verticales ( 4 pieds) et horizontales (traverses). 
- ces socles sont orientés : pont (" ouvert ») de face, et cadre sur le côté,

- ce cadre latéral permet de prévoir sous celui-ci-une plaque métallique, à même de répartir le poids des œuvres lourdes sans détériorer le parquet Versailles.

Ce vocabulaire prototype, dessiné pour le Musée Rodin, est décliné sur toute une série de supports, de natures et tailles différentes : socle, table, vitrine haute ou basse, console... Typologiquement, ils se déclinent suivant 4 hauteurs, 4 largeurs de base et des dimensions spécifiques pour les grands supports ou consoles murales. Quelques supports spécifiques sont définis pour les œuvres particulières.

Un point fondamental dans le projet de mobilier est l'absence de climatisation, entraînant des variations de températures et d'hygrométrie rapides et fortes. Or, un bois massif est un matériau vivant susceptible de se déforme. Le support bois massif habille donc de manière invisible une structure primaire en profilés d'aluminium, la seule à même :

- de n'avoir aucune déformation et assurer une stabilité dans le temps, quelles que soient les variations des conditions climatiques,

- de transmettre sans déformation le poids d'œuvres qui peuvent être très lourdes $(300-400 \mathrm{~kg})$,

- d'assurer la finesse et l'élégance des supports malgré des poids très importants et des portées pouvant aller jusqu'à $1,80 \mathrm{~m}$ sans support intermédiaire.

Ces mobiliers présentent soit directement les œuvres, soit sont supports de vitrines, le support étant identique pour un socle ou pour une vitrine.

Les vitrines au-dessus des socles sont conçues pour être les plus discrètes possibles : sans structure métallique, en verre extra blanc collé à coupe d'onglet, ouvrant d'accès par pivot invisible, serrure invisible, etc.

Ces mobiliers sont en réalité très techniques: réglage de l'altimétrie, de horizontalité, étanchéité, climatisation pour certaines vitrines, "meccano» interchangeable, etc.

Dans le Musée Rodin, comme dans la plupart des musées actuellement, les œuvres peuvent assez souvent changer de place. Les mobiliers en partie centrale ne sont donc pas équipés d'éclairage en interne (celui-ci aurait nécessité d'une part de percer le parquet Versailles classé, et ensuite de figer bien avant la pose des œuvres l'ensemble des positions des mobiliers et donc des œuvres, ce qui n'était pas envisageable).

De même, le positionnement fin des supports et donc des œuvres ne peut s'effectuer qu'une fois celles-ci en place, et les rapports entre elles réellement évalués sur place. Pour ce faire, l'ensemble des mobiliers est transportable par transpalette, quel que soit le type de support.

Les cloches en verre ont été proscrites, au bénéfice de vitrines en totalité équipées d'ouvrants, et donc permettant de manière très simple les mouvements d'œuvres sans faire obligatoirement appel à une entreprise spécialisée.

Pour finir, dans certaines salles (hall, "Rodin à Biron ", impressionnisme) un mobilier ancien, contemporain de Rodin lui-même est présenté (sellettes, vitrine, meubles, fauteuil, caisses...).

\section{Aménager des salles de musée}

À partir de ce vocabulaire homogène, il s'agit d'éviter toute monotonie. Il s'agit aussi de gérer la densité, la présentation passant de 380 à 600 œuvres (soit plus de $50 \%$ d'augmentation). Pour ce faire, quatre principes d'organisation des salles ont été retenus :

- au centre des salles et vers les baies, les œuvres ont été limitées en nombre, autant que possible de grandes dimensions, majeures, hors vitrine, ou en grandes vitrines ou tables de regroupement d'une ou plusieurs œuvres, afin de créer un effet significatif en entrant dans la salle (effet " Waouhh") 
(fig. 2). Cette limitation en occupation de la salle se fait au bénéfice du confort et de la fluidité de la visite en salle, et surtout de la contemplation des œuvres avec des vues dégagées.

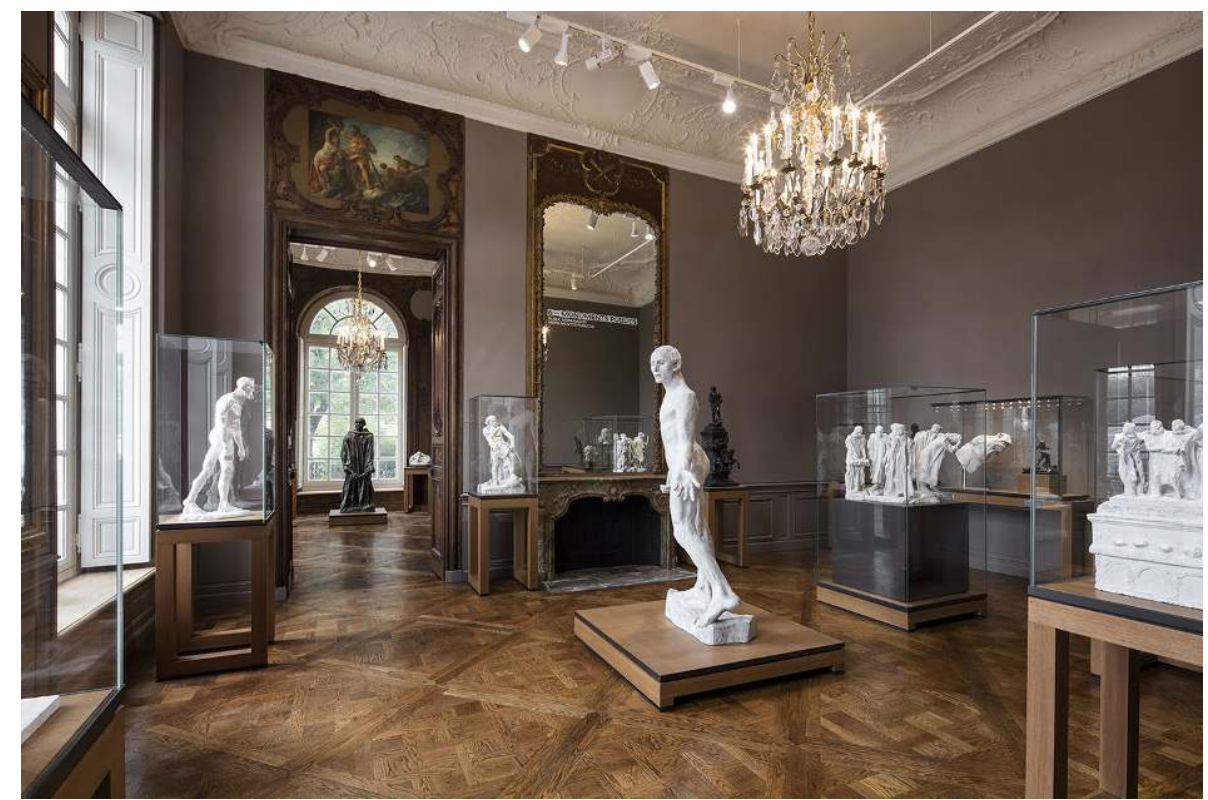

Fig. 2

Vue de la salle 6 Monuments publics » Paris, Hôtel Biron
(c) Patrick Tournebœuf / OPPIC / Tendance Floue

En corollaire, en périphérie de salle les œuvres sont plus denses : de longues consoles murales rassemblent plusieurs œuvres, hors ou dans les vitrines, avec des sur-tablettes hiérarchisant les présentations :

- le principe général est que les mobiliers muséographiques sont implantés suivant une trame orthogonale calée sur les baies, portes et axe des salles et entre les mobiliers eux-mêmes, avec des vis-à-vis, continuités ou décalages finement étudiés. Mais, de salle en salle, des organisations spécifiques sont mises en œuvre, par leur position dans l'hôtel (axes, angles), leur forme (ovale) ou leur thème ("Rodin à Biron») (fig. 3 et 4). Ces dispositifs muséographiques particuliers permettent de donner un rythme à la présentation. De même, on pourra passer d'une salle sans vitrine à une salle entièrement équipée de vitrines.

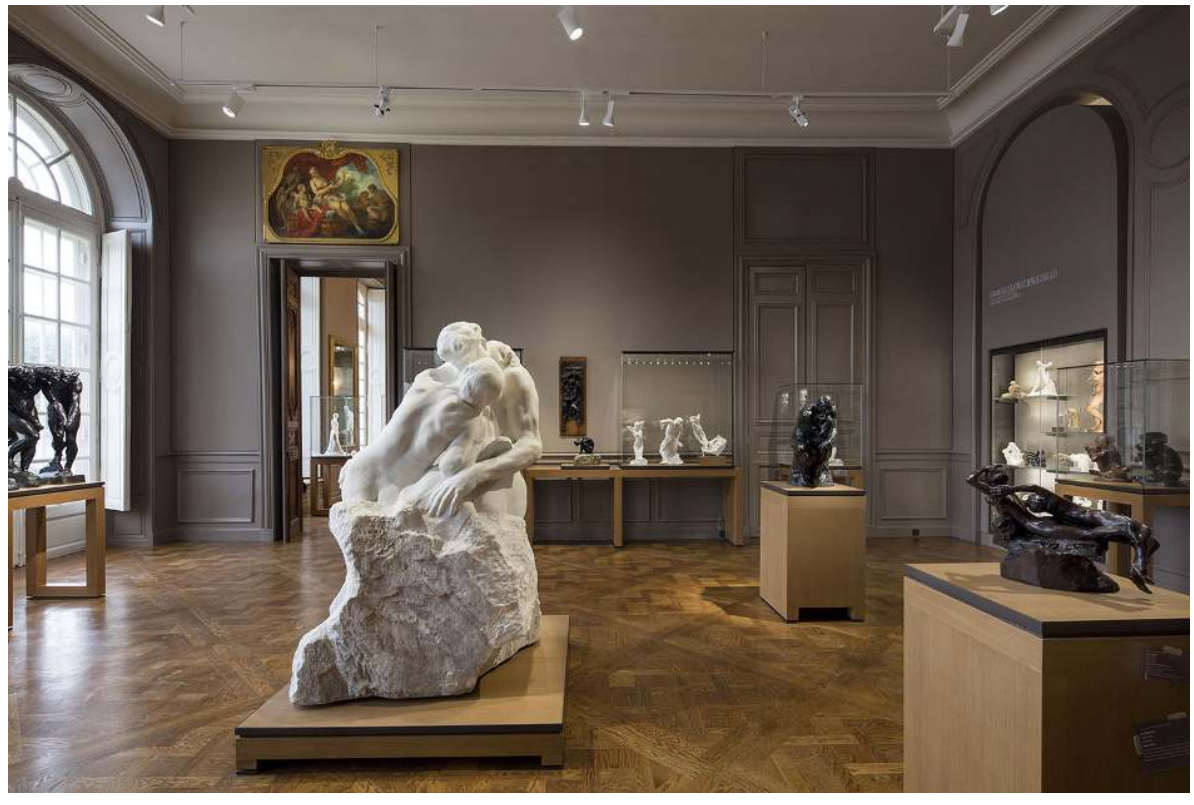

Fig. 3

Vue de la salle 5 La porte de l'Enfer » ebœuf / OPPIC / Tendance Floue
- une dominante est définie par salle pour lui donner une identité propre, établie sur la base de ses typologies de mobilier majoritaires, afin d'éviter la juxtaposition de trop de mobiliers différents dans une même salle (voir fig. 2). Une hauteur de mobilier est ainsi définie salle par salle. 


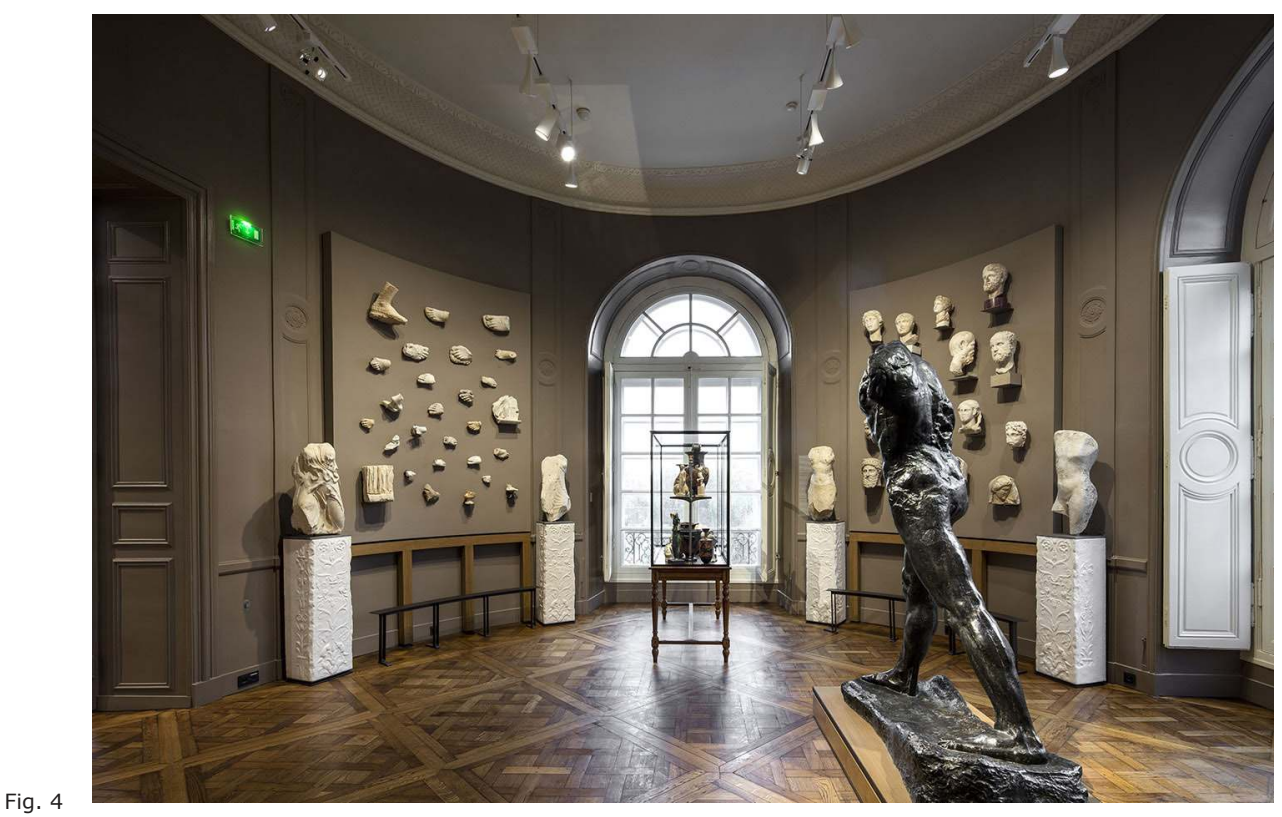

Vue de la salle 17 Rodin et I'Antique » Paris, Hôtel Biron (c) Patrick Tournebœuf / OPPIC / Tendance Floue

La présentation varie ainsi d'une salle à l'autre, par des alternances de densité et de positionnement, de symétrie et de dissymétrie, entre " ordre " et « désordre »:

- un certain nombre d'œuvres sont placées soit en appel et axées, soit en perspective. En appel et dans l'axe, elles marquent un changement de direction dans le circuit, et appuient une perspective (fig. 5). De salle en salle, elles se perçoivent comme une "incitation à avancer", dans une perspective ouverte : décalées, elles laissent libre le passage et la vue dégagée vers le fond de la perspective, positionnées vers les baies pour bénéficier au mieux de l'éclairage naturel.

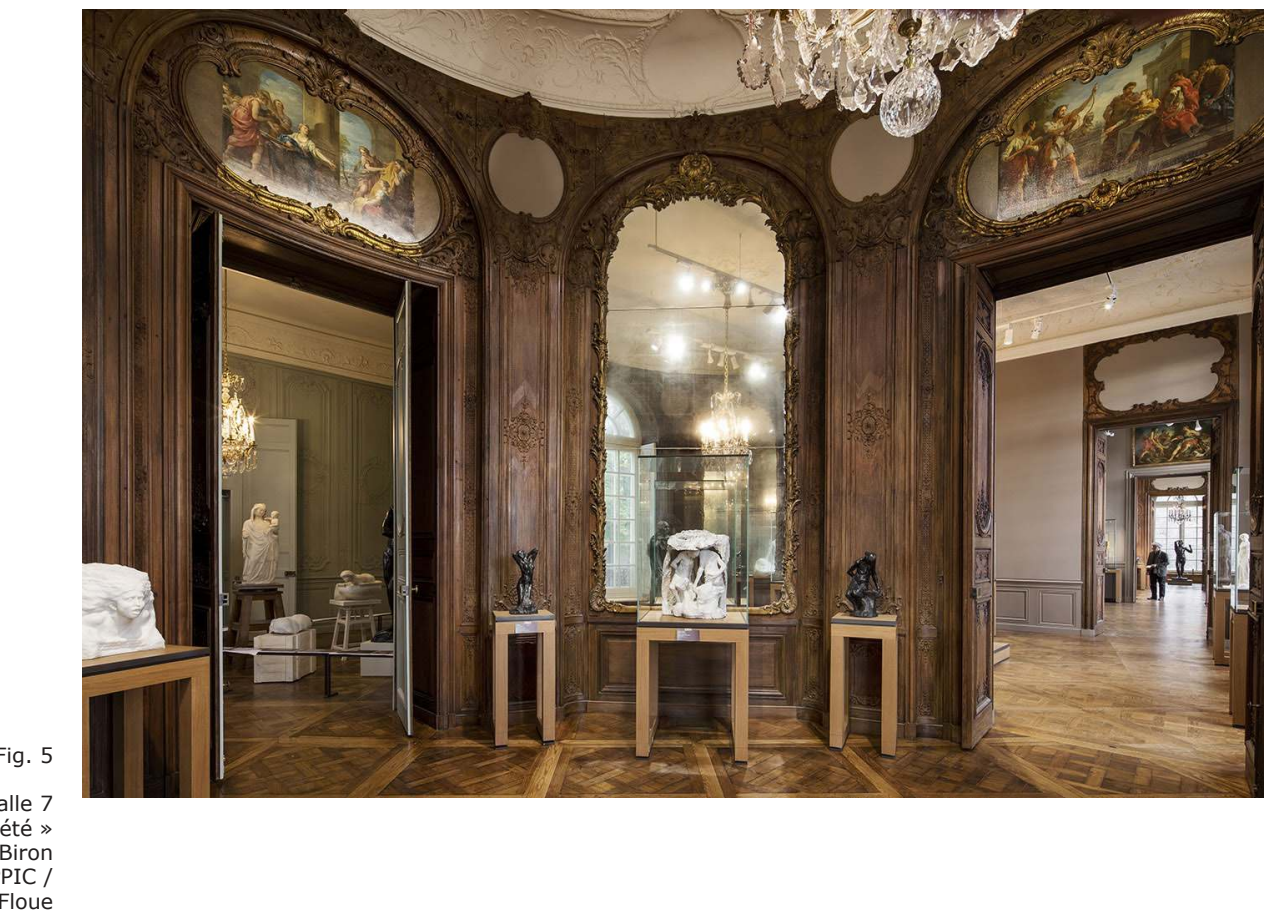




\section{3. Éclairer la sculpture}

La sculpture, c'est la lumière. Le traitement de la lumière occupe donc une place prépondérante dans la réflexion sur le nouveau Musée Rodin. Elle est fondatrice du projet muséographique, et indispensable pour bien voir la sculpture et jouer avec les volumes.

La magie de l'hôtel Biron est due à l'abondante lumière pénétrant par ses larges baies vitrées (accentuée par les reflets des miroirs) et cette vue imprenable sur le jardin, vaste musée à ciel ouvert, où les œuvres de Rodin sont révélées en permanence par la lumière du jour changeante.

Tout le projet de la lumière artificielle vise à préserver au maximum cet apport naturel. Il se fait le relais de l'éclairage naturel et permet de préserver un éclairage optimal des œuvres au gré des variations du temps, des saisons et des heures. Il appuie le propos muséologique et muséographique et aide à la hiérarchisation des œuvres.

Ce nouvel éclairage innovant et performant, est fondé sur la technologie LED, pilotée par un système informatique (protocole Dali) programmé pour régler l'intensité et/ou la température de couleur œuvre par œuvre, projecteur par projecteur, dans la salle à partir d'une tablette. Ce réglage "statique " est complété par un réglage "dynamique » qui varie salle par salle (à partir de sondes en plafond), qui permet de faire varier l'intensité en fonction de la lumière naturelle mesurée dans la salle, afin de préserver au mieux ce rapport subtil entre lumière naturelle et lumière artificielle tout au long de la journée et des saisons. Plusieurs scénarios sont ou seront donc installés, hiver ou été ; des scénarios plus spécifiques peuvent être imaginés : événement, visite à la bougie... Ces scénarios intègrent en outre les heures d'ouverture et de fermeture du musée, les jours de fermeture, etc. Ce principe d'éclairage sera installé pour l'une des toutes premières fois dans un musée en Europe.

\section{Bien voir la sculpture, colorer l'arrière-plan}

Le projet utilise, avec le partenariat de Farrow \& Ball, une gamme de gris colorés pour les murs des salles (hors boiseries), sur les principes suivants :

$-s$ 'inspirer des teintes anciennes découvertes lors des sondages, tenant compte des teintes connues lors de l'ouverture du musée par Rodin lui-même (photos et textes),

- retenir des densités moyennes, afin de révéler autant les marbres et les plâtres (blancs) que les bronzes (sombres),

- créer un rythme par les changements de couleur, marquant le passage d'une section à une autre, mais en limitant le nombre de teintes pour assurer à la fois une maintenance aisée et une homogénéité de l'ensemble,

- atténuer au rez-de-chaussée les ruptures de teinte et de densité entre les murs des salles sans boiseries et celles à lambris (boiseries sombres), avec une création avec F\&B d'une teinte spécifique pour le Musée Rodin, le "Biron Grey " (hors catalogue),

- marquer en étage la spécificité des rotondes Est et Ouest à la fois pour des raisons « architecturales " et pour des raisons de programme (Alma à l'Est, et Antiques à l'Ouest),

- recréer pour le hall et l'escalier d'honneur un ensemble cohérent avec la pierre existante avec une teinte " pierre » chaleureuse.

Les plafonds sont traités simplement, de manière contemporaine, monochromes en évitant la teinte différenciée des guirlandes et autres décors, en blanc cassé gris chaud. 


\section{Les acteurs}

Maîtrise d'ouvrage :

Ministère de la Culture et de la Communication/Établissement Public du Musée Rodin (EPMR)

Maîtrise d'ouvrage déléguée :

Opérateur du Patrimoine et des Projets Immobiliers de la Culture (OPPIC)

OPC : SCTB/CSPS : BECS/Bureau de contrôle : SOCOTEC

Maîtrise d'œuvre muséographie et mise aux normes :

SARL Atelier de l'île architectes paysagistes: Dominique BRARD, Olivier LE BRAS et Marc QUELEN

Mandataire : Dominique BRARD architecte, co-gérant

Chef de Projet : Marcel DAVIDSE

Éclairagiste : Agence Stéphanie DANIEL

Économiste : SORETEC

Bet électricité : BLEUSE

Entreprises muséographie, aménagement et mise en sécurité :

Aménagement, plâtrerie, menuiserie, plomberie : MPI Action

Peinture : DUVAL \& MAULER/peintures FARROW \& BALL

Mobilier muséographique : MEYVAERT

Courants forts et courants faibles : DERICHEBOURG

Éclairage Muséographique : BOUYGUES ES/matériel ZUMTOBEL

Maîtrise d'œuvre monuments historiques : Richard DUPLAT ACMH

Entreprises restauration des parquets et confortation des planchers :

Installations de chantier : Buhr-Ferrier-Gosse

Maçonnerie : H Chevalier

Plâtrerie, gypserie : SOE Stuc et Staff

Menuiserie, ébénisterie : Asselin SAS

Charpente, reprise structurelle : Cruard

Peinture, peinture décorative : Meriguet-Carrere

Lustrerie : Vallux

Entreprises restauration des menuiseries extérieures, parcours de couverture :

Menuiserie : Asselin

Métallerie : Cruard

Peinture : Duval \& Mauler

Maçonnerie : MPR

Couverture : UTB

Entreprises Aménagement de la cour intérieure :

Maçonnerie : MPR

Menuiserie : Asselin

Métallerie : Roussot

Peinture : Duval \& Mauler

Espaces verts : Val d'Oise Jardins

Électricité : Bouygues Énergie

Entreprises Aménagement des combles :

Maçonnerie : H Chevalier

Charpente, reprise structurelle : Cruard

Menuiserie, ébénisterie : Asselin SAS

Cloison, doublage, faux-plafond : Marc Meunier

Peinture, revêtement de sols souples : Trouve Leclaire

Électricité : Satelec 
Couverture, plomberie : UTB

Photographies : P. Tourneboeuf/Oppic

\section{L'auteur}

Attaché à la diversité des exercices de la profession d'architecte et au travail pluridisciplinaire, Dominique Brard a une expérience affirmée de la muséographie permanente et de l'exposition temporaire. Exerçant dans un atelier d'architecture ou il allie des compétences en muséographie, en paysage, en conseil ou en urbanisme, avec des partenaires réguliers d'autres disciplines, il intervient à différents stades de leur mise en œuvre : maîtrise d'ouvrage, programmation architecturale, muséologie, maîtrise d'œuvre... Parmi ses réalisations récentes dans le domaine, on peut citer le musée des Beaux-Arts de Pont-Aven (livraison prévue pour 2016), la muséographie du Pavillon Amont du musée d'Orsay en 2011, le réaménagement du département des Antiquités égyptiennes du musée du Louvre (1998), mais également des scénographies : l'exposition Silent Partner au musée Bourdelle en 2015, David d'Angers dessinateur à la galerie David d'Angers en 2014.

\section{The author}

Devoted to the diversity of exercising the profession of architect and multidisciplinary work, Dominique Brard has experience in permanent museography and temporary exhibitions. Working in an architecture studio where he brings together skills in museography, landscaping, consulting and town planning, with regular partners from other disciplines, he intervenes at different stages of their implementation, including contracting, architectural programming, museology and project management. His recent works in the field include the Musée des Beaux-Arts de Pont-Aven (scheduled for completion in 2016), the museography of the Pavillon Amont of the Musée d'Orsay in 2011, the renovation of the department of Egyptian Antiquities at the Musée du Louvre (1998), and the exhibition designs for the exhibition Silent Partner at the Musée Bourdelle in 2015 and David d'Angers dessinateur at the Galerie David d'Angers in 2014. 